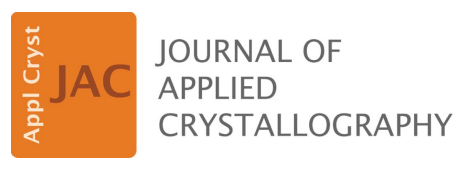

ISSN 1600-5767

Received 6 February 2019

Edited by G. J. McIntyre, Australian Nuclear Science and Technology Organisation, Lucas Heights, Australia

Keywords: Inorganic Crystal Structure Database; ICSD; theoretical structures; standardization; classification.

Supporting information: this article has supporting information at journals.iucr.org/j

\section{Recent developments in the Inorganic Crystal Structure Database: theoretical crystal structure data and related features}

\author{
D. Zagorac, ${ }^{a, b *}$ H. Müller, ${ }^{c}$ S. Ruehl, ${ }^{c *}$ J. Zagorac ${ }^{a, b}$ and S. Rehme ${ }^{c}$
}

Accepted 12 July 2019

\author{
${ }^{\mathbf{a}}$ Technicum Scientific Publishing, Stuttgart, Germany, ${ }^{\mathbf{b}}$ Institute of Nuclear Sciences Vinča, Materials Science Laboratory, \\ Belgrade University, Belgrade, Serbia, and ${ }^{\mathbf{C}} \mathrm{FIZ}$ Karlsruhe - Leibniz Institute for Information Infrastructure, Karlsruhe, \\ Germany. ${ }^{*}$ Correspondence e-mail: editor@opentechnicum.com, stephan.ruehl@fiz-karlsruhe.de
}

The Inorganic Crystal Structure Database (ICSD) is the world's largest database of fully evaluated and published crystal structure data, mostly obtained from experimental results. However, the purely experimental approach is no longer the only route to discover new compounds and structures. In the past few decades, numerous computational methods for simulating and predicting structures of inorganic solids have emerged, creating large numbers of theoretical crystal data. In order to take account of these new developments the scope of the ICSD was extended in 2017 to include theoretical structures which are published in peer-reviewed journals. Each theoretical structure has been carefully evaluated, and the resulting CIF has been extended and standardized. Furthermore, a first classification of theoretical data in the ICSD is presented, including additional categories used for comparison of experimental and theoretical information.

\section{Introduction}

The Inorganic Crystal Structure Database (ICSD) contains an almost exhaustive list of known inorganic crystal structures published since 1913 (Bergerhoff \& Brown, 1987; Belsky et al., 2002). In particular, the database provides information on structural data of pure elements, minerals, metals and intermetallic compounds. In order to be included in the database, a structure has to be fully characterized, the atomic coordinates determined and the composition fully specified. A typical entry includes, inter alia, the chemical name, formula, unit cell, space group, complete atomic parameters (including atomic displacement parameters), site occupation factors, title, authors and literature citation. In addition to the published data, many items are added through expert evaluation or are generated by computer programs, such as the Wyckoff sequence, molecular formula and weight, $\mathrm{ANX}^{\mathbf{1}}$ formula, mineral group etc. (Buchsbaum et al., 2010). Of course, full bibliographic information is also included; for newer entries often even the abstract is provided.

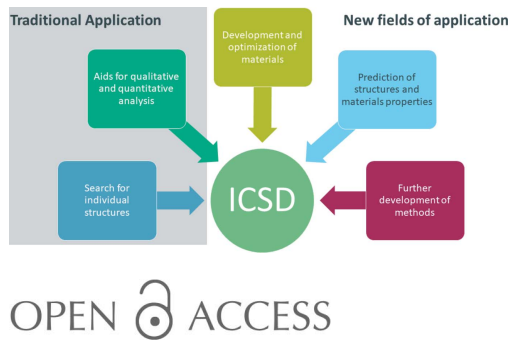

\footnotetext{
${ }^{1}$ The ANX formula is a simple classification for structures based on the oxidation states of the elements involved. Elements with a positive oxidation state are identified by the first letters of the alphabet $\mathrm{A}-\mathrm{M}$, elements with a negative oxidation state by the last letters of the alphabet $\mathrm{S}-\mathrm{Z}$ and elements with an oxidation state of 0 by the letters $\mathrm{N}-\mathrm{R}$. The letters are sorted by increasing index (AB2X4, not A2BX4). Structures containing more than four positive, three negative or three neutral atomic types are not considered. The ANX formula is only calculated for completely determined structures and hydrogen atoms are not considered.
} 
All crystal structures contained in the database have been carefully evaluated and checked for quality related to formal errors and scientific accuracy by our expert editorial team. We continuously extract and abstract the original data from over 80 leading scientific journals and an additional 1300 scientific journals. The ICSD is updated twice a year, each time adding approximately 4000 new records. As the size of the ICSD has grown over time, we have continuously enhanced the quality of our data. At present (2018.2 release), the ICSD contains more than 200000 entries, including 2902 crystal structures of the elements, 38506 records for binary compounds, 73048 records for ternary compounds, and 73688 records for quarternary and quintenary compounds. About 159000 entries (80\%) have been assigned to one of 9015 structure types (Allmann \& Hinek, 2007). The remaining $20 \%$ of entries are not assigned to any existing structure type, as such compounds would be individual compounds with a new structure type of their own and, according to our definition, a structure type has to contain at least two compounds.

In the beginnings of the ICSD, the focus was merely on collecting and editing data. The data available in the literature were identified and examined according to defined quality criteria. In the meantime, the ICSD has evolved from a mere collection of data into a versatile tool for research and materials science (Fig. 1). Pure structure information is combined with information on physical-chemical properties and measurement methods. This means that the data can be used more universally. Last but not least, as a result of discussions about data mining and the application of semantic tools, article- and structure-related keywords (not necessarily identical to the author keywords, which are often too general) and the abstracts contained in the articles have been included in the database in recent years. Starting with the publication year 2015, theoretical (calculated) structures have also been recorded in the ICSD.

A database needs to cover several essential aspects in order to be useful (Buchsbaum et al., 2010). The first aspect is the comparability of data. For crystallographic data this is easy as the comparability is already based on the principles of crystallography itself and further enforced by standardizing all crystal structures for better comparison. A generally accepted format is even defined for the exchange of crystallographic information (crystallographic information file - CIF; Hall, 1991; Hall \& Spadaccini, 1994). The second important aspect is the completeness of data. Statistical interpretations based only on a small subset will probably not produce results with a high level of significance. The last and most decisive factor is the quality of the data. Unreliable data can only lead to unreliable results. For the ICSD, in the case of distinctive features the author is contacted or a remark is set.

\section{Comparison with other crystal-structure-based databases}

In addition to the ICSD, there are several other commercial and non-commercial structure-based databases. Among the commercial databases, the Cambridge Structural Database (CSD; Groom et al., 2016) published by the Cambridge Crystallographic Data Centre (CCDC), the various powder diffraction file (PDF; ICDD, 2018) databases of the International Centre for Diffraction Data, Pearson's Crystal Data (Villars \& Cenzual, 2018), CrystMet (White et al., 2002) and AtomWork-Adv (NIMS, 2018) are worth mentioning.

The bandwidth of non-commercial databases is very wide, ranging from the Protein Data Bank (Berman et al., 2000), which specializes in proteins and nucleic acids, to the generally oriented Crystallography Open Database (Gražulis et al., 2012) and the American Mineralogist Crystal Structure Database (Downs \& Hall-Wallace, 2003), to a large number of databases for calculated structures (Curtarolo et al., 2012; Jain et al., 2013; Saal et al., 2013; Draxl \& Scheffler, 2018; http:// openmaterialsdb.se/; Ortiz et al., 2009).

Table 1 bundles the most important information of the different databases and allows quick comparison.

Apart from the differences in domain coverage and some special functionalities, the completeness and consistency of the experimental data offered is certainly the greatest in the commercial services. For the mentioned purposes of data mining these databases are most suitable.

FIZ Karlsruhe has been cooperating with the CCDC since 2017 and provides a joint crystal structure depository in which all crystal structures of the CSD, the ICSD and the previous

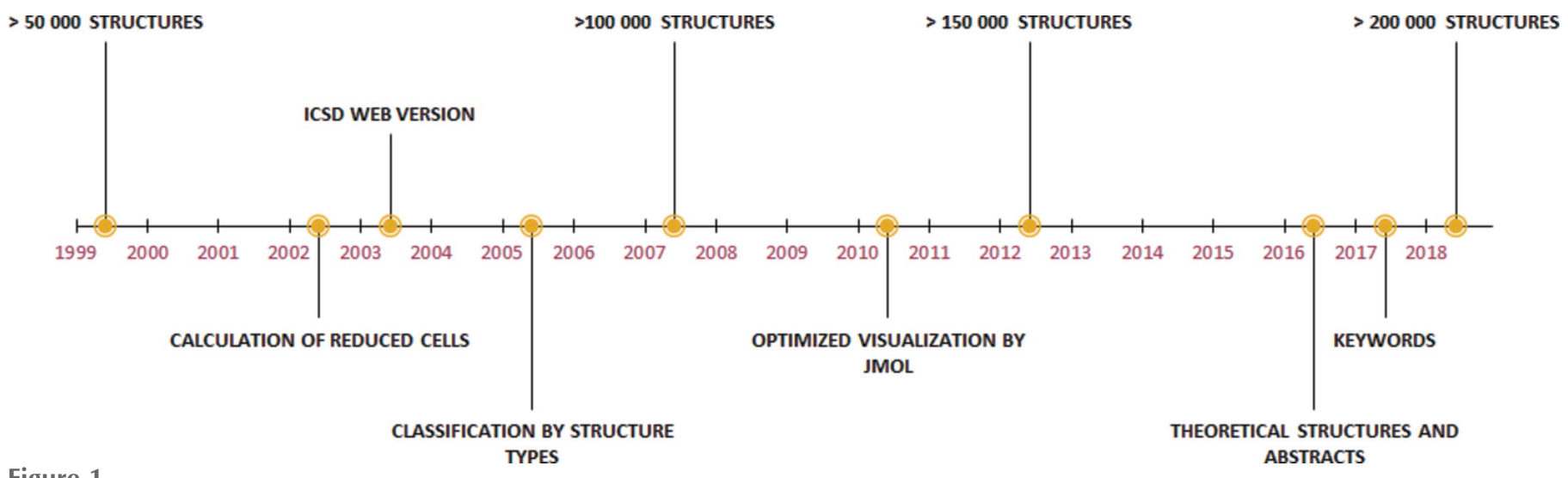

Figure 1

ICSD timeline. 
Table 1

Comparison of databases containing experimental and/or theoretical crystal structures.

\begin{tabular}{|c|c|c|c|}
\hline & No. of entries & Content & Remarks \\
\hline ICSD & $\sim 210000$ & Inorganic and metal-organic compounds & $\begin{array}{l}\text { Commercial, experimental and calculated } \\
\text { structures, material properties }\end{array}$ \\
\hline CSD & $\sim 1000000$ & Organic and metal-organic compounds & Commercial, experimental structures \\
\hline PDF & $\sim 410000(\mathrm{PDF}-4+)$ & Inorganic and organic compounds & $\begin{array}{l}\text { Commercial, powder data, not all entries include } \\
\text { atomic coordinates }\end{array}$ \\
\hline Pearson's Crystal Data & $\sim 319000$ & Inorganic compounds & $\begin{array}{l}\text { Commercial, experimental structures, not all } \\
\text { entries include atomic coordinates }\end{array}$ \\
\hline CrystMet & $\sim 180000$ & Inorganic compounds & Commercial, experimental structures \\
\hline AtomWorks-Adv & $\sim 300000$ & Inorganic compounds & $\begin{array}{l}\text { Commercial, experimental structures and mate- } \\
\text { rial properties, not all entries include atomic } \\
\text { coordinates }\end{array}$ \\
\hline Protein Data Bank & $\sim 150000$ & Proteins, nucleic acids & Open access, experimental structures \\
\hline Crystallography Open Database & $\sim 400000$ & Inorganic and organic compounds & Open access, experimental structures \\
\hline $\begin{array}{l}\text { American Mineralogist Crystal } \\
\text { Structure Database }\end{array}$ & $\sim 20000$ & Only minerals & Open access, experimental structures \\
\hline Aflowlib & $\sim 2800000$ & $\begin{array}{l}\sim 350000 \text { binaries, } \sim 1900000 \text { ternaries and } \\
\quad \sim 450000 \text { quaternaries }\end{array}$ & $\begin{array}{l}\text { Open access, calculated structures and material } \\
\text { properties, all calculated using Aflow }\end{array}$ \\
\hline Materials Project & Unknown & $\begin{array}{l}\sim 530000 \text { nano-porous compounds, } \sim 130000 \\
\text { inorganic compounds }\end{array}$ & $\begin{array}{l}\text { Open access, calculated structures and material } \\
\text { properties }\end{array}$ \\
\hline Open Quantum Materials Database & $\sim 560000$ & Inorganic compounds & $\begin{array}{l}\text { Open access, calculated structures and material } \\
\text { properties }\end{array}$ \\
\hline Nomad & $\sim 50000000$ & Inorganic and organic compounds & $\begin{array}{l}\text { Open access, calculated structures and material } \\
\text { properties }\end{array}$ \\
\hline Open Materials Database & $\sim 200000$ & Inorganic and organic compounds & $\begin{array}{l}\text { Open access, calculated structures and material } \\
\text { properties }\end{array}$ \\
\hline Electronic Structure Project & $\sim 60000$ & Inorganic and organic compounds & Open access, calculated structures \\
\hline
\end{tabular}

separate crystal structure depositories are stored and freely accessible - however, the search options are very limited and the export of crystal structures is also limited. Further cooperations are planned and will bring the two databases even closer together.

\section{Keywords in the ICSD}

Compounds with defined material properties can be searched in the ICSD owing to the introduction of keywords. Search results based on titles and abstracts are often limited, because they present the author's priorities. Specific keywords are assigned according to the content of the article and are therefore more precise. In most cases an article already contains author keywords. These, however, are often too general (e.g. 'crystal structure') and not suited for searching for defined properties and methods. The keywords in the ICSD are assigned according to a defined thesaurus and thus standardized. Additional free-text entries can be made in exceptional cases.

At the beginning of the assignment of keywords, about 20000 keywords, mainly from the fields of magnetism and spectroscopy, were assigned to about 6000 journal articles from the running production within the year 2018.

As regards the frequency distribution of the individual keywords, almost 280 keywords (as per January 2019) were considered relevant and made available to the users as a first ad hoc list: not least in order to receive feedback from the users as soon as possible.

The current ICSD thesaurus is not static but is continuously extended. Depending on the development of the discipline we will see where a deeper indexing will be required in the future. Among the next steps - besides the desired feedback from the community - could be a comparison with recognized thesauri and ontologies from science and technology in order to close gaps in the hierarchic structure of the ICSD. We also plan to employ data mining procedures in order to index ICSD structures retrospectively on the basis of titles and abstracts.

ICSD keywords describe material properties, analysis methods used or technical fields of application (Fig. 2).

In particular, material properties are further classified as magnetic properties, electrical properties, optical properties, mechanical properties, thermal properties, physicochemical properties and dielectric properties (Fig. 2). Each of these rather broad descriptions is further split into more detailed keywords fully searchable in the ICSD, e.g. magnetic properties with magnetic susceptibility or ferromagnetism, electrical properties with superconductivity or piezoelectricity, and so on (for more details see the supporting information).

Similarly, applied methods are also classified into spectroscopic methods, thermometry, calculations, electrochemistry, magnetometry, microscopy, crystal structure, chemical composition $^{2}$ and synthesis, and for each of them specific keywords are assigned which are frequently encountered in scientific and industrial work (for details $c f$. supporting information). Technical application is described by the keywords optoelectronics, energy, spintronics, environmental properties, catalysis, zeolites and biology. As in previous cases, a set of more detailed keywords has been assigned, fully searchable in

\footnotetext{
${ }^{2}$ Not included in Fig. 2; for a current list of keywords see https:// icsd.products.fiz-karlsruhe.de/en/howuse/how-use.
} 
the ICSD, i.e. for optics keywords like nonlinear optics (NLO) materials or light emitting diode (LED) technology, for energy keywords like solar cells or batteries, and so on (cf. supporting
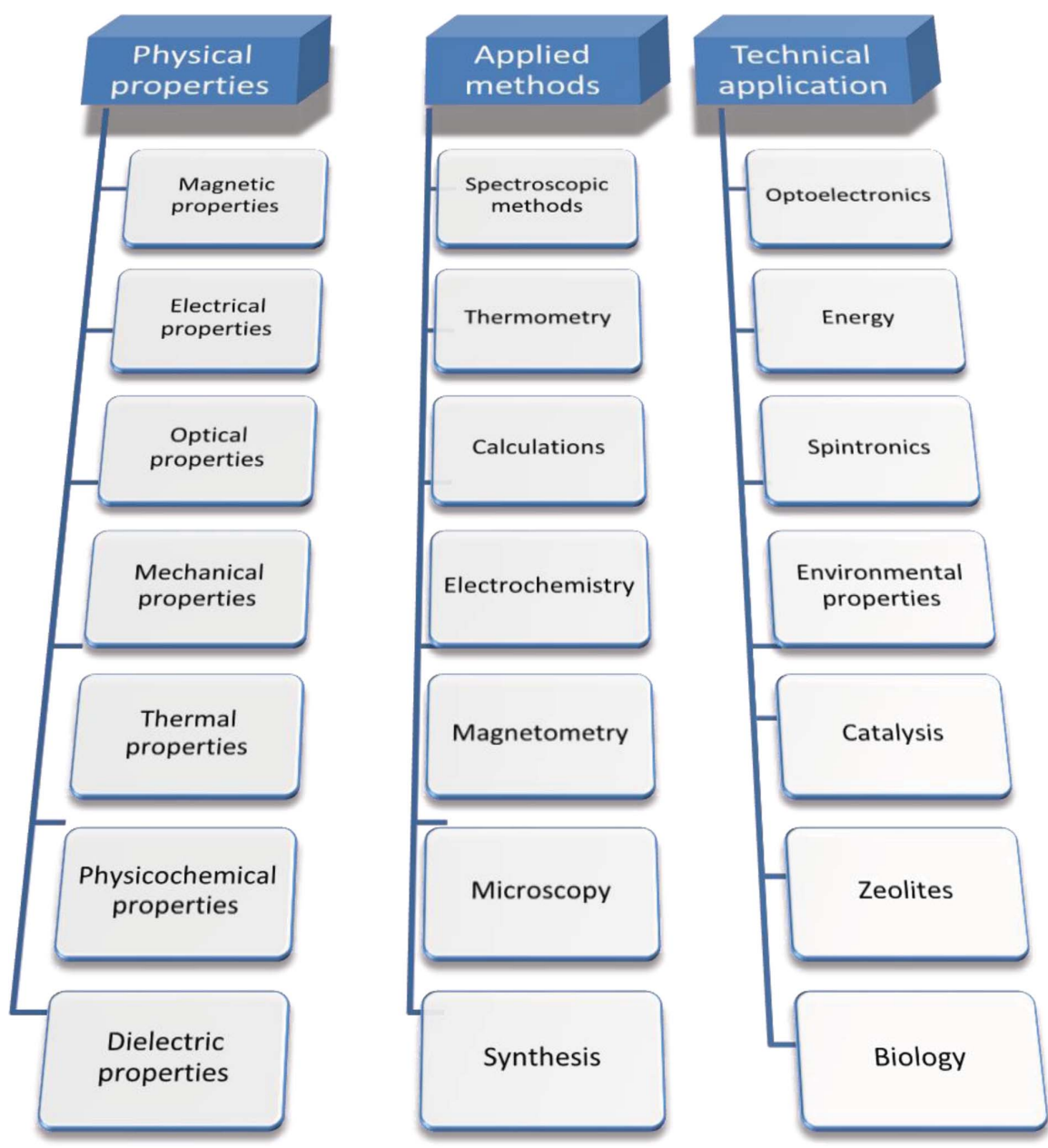

Figure 2

Set of predefined keywords standardized according to physical properties of materials, applied methods and technical application, fully searchable in the ICSD. For the full list of the standardized keywords see the supporting information.

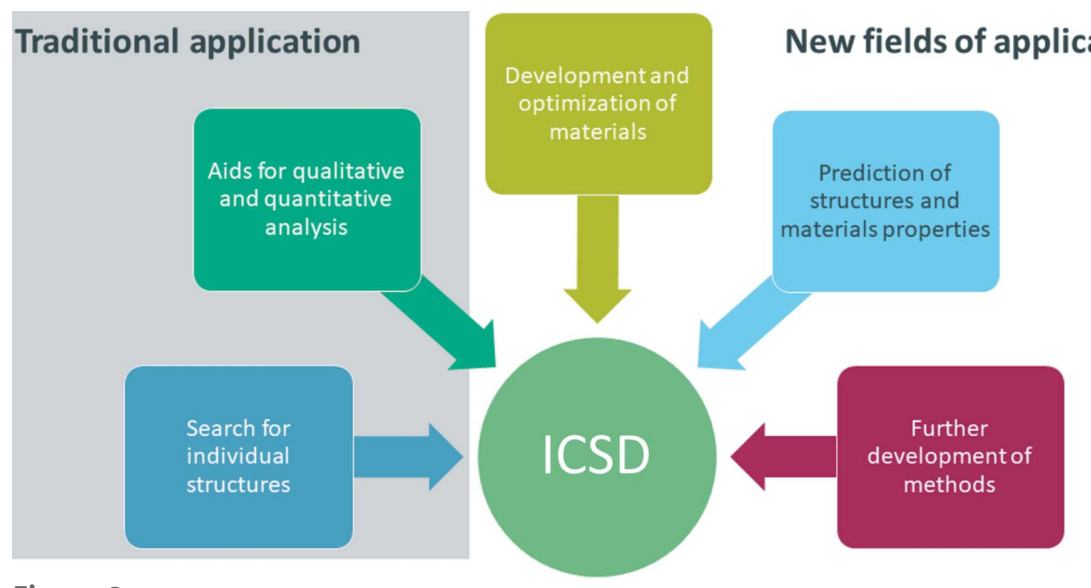

Figure 3

ICSD application graph, going from traditional applications such as searches for individual structures and using them in qualitative or quantitative analysis, to new fields of application, where the data are used to develop or optimize new materials following either the classical synthesis approach or the more modern in silico approach. information). In addition to standardized keywords, a freetext keywords search is available in the ICSD, e.g. in order to search the ICSD for nanostructures, the user needs to type free-text nano (e.g. Káňa et al., 2016; Miao et al., 2016).

In summary, the use of keywords combined with, for example, chemical (elements) or structural (structure types) information easily enables searches for special materials like superconductors or piezoelectric materials or technical applications like solar cells or solid electrolytes.

\section{Theoretical structures}

\subsection{Standardization of theoretical crystal structures in the ICSD}

More and more tailor-made materials with predefined properties are being produced (Butler et al., 2016). Predicting material properties or synthesizing special properties can save time-consuming and expensive work in the laboratory, and this is now possible because of the availability of high computing power and improved computer programs (Curtarolo et al., 2013). To develop new materials, it is usually necessary to use structure information from existing and already measured compounds contained in suitable databases, e.g. the ICSD. Optimizing the available parameters or comparing measured and calculated results can then lead to new conclusions, as summarized in Fig. 3.

The ICSD is already extensively used in data mining and in computational chemistry. The traditional approach in materials research of first synthesizing new compounds and then checking their properties is rather time consuming and quite expensive. One can already observe a strong tendency to shift materials research from the traditional synthesisoriented approach to a more theoryoriented approach. On the other hand there are numerous problems with available theoretical data (lack of file format standardization, the variety of methods/ codes etc.), and perhaps the major problem is the huge quantity of calculated data with a broad variety of quality.

In order to tackle these problems, we have performed data standardization of the theoretical crystal structures. Data standardization is the critical process of 
bringing data into a common format, implementing and developing technical standards, and helping to maximize the quality of the data. In particular, a set of selection criteria has been developed in order to standardize theoretical crystal structure data. We have three major criteria for the selection of theoretical structures:

(a) publication criterion;

(b) total energy criterion;

(c) multiple methods criterion.

The first criterion for selection of theoretical structures is publication in a peer-reviewed journal. In this way, we are able to discard a large quantity of theoretical data which are unpublished and stored in various databases, with unknown origin or quality. However, this selection criterion involves careful evaluation and a great amount of manual work through inspection of individual research papers in order to ensure high quality of the extracted theoretical structures. The second criterion includes total energy ranking of the theoretical structures. In principle, theoretical structures which have low total energy are considered to be close to the equilibrium structure and suitable for storing in the ICSD. In addition, theoretical structures that are affected by external conditions (pressure, temperature, magnetic field etc.) are deposited, and this information is provided in the corresponding CIF. Similarly, theoretical structures with negative formation energies are considered suitable. In this way a large number of highenergy and extremely metastable theoretical structures, which are not likely to be synthesized, are excluded. The final criterion is applicable when multiple theoretical methods have been applied to calculate the same starting structure. In such cases, the theoretical method which delivers data closest to the corresponding experimental results is chosen for storing in the ICSD, while other methods applied are only noted as a comment.

Otherwise, theoretical data are completely coherent with experimental data in the CIF: for example, each record contains information on compounds which have no $\mathrm{C}-\mathrm{C}$ and/

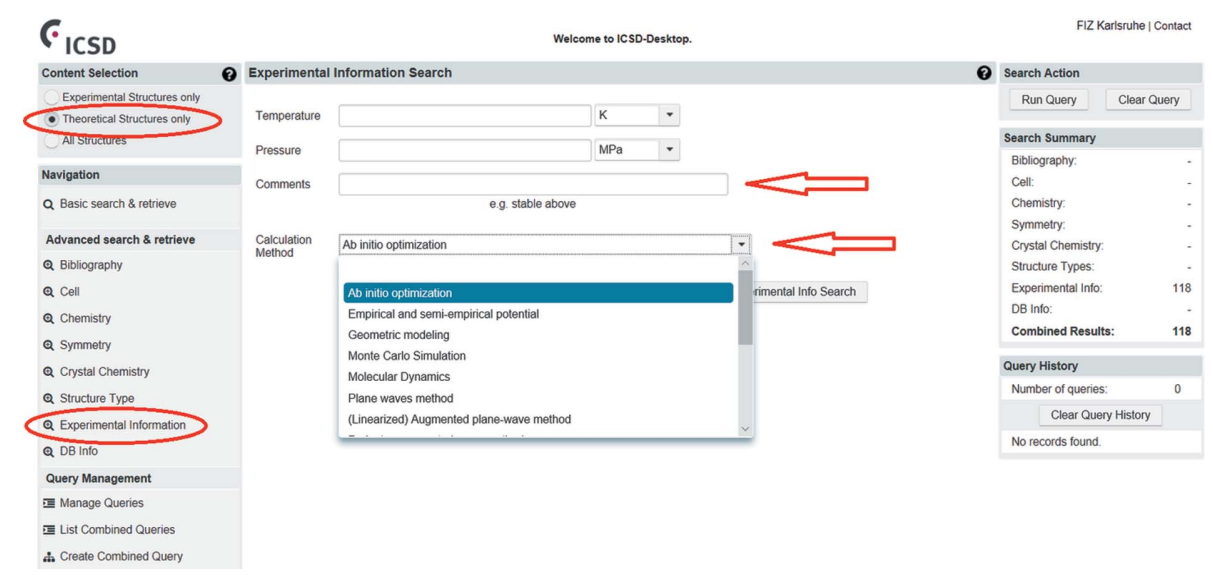

Figure 4

In the 'Content Selection' the user can choose 'Theoretical Structures only' (upper left corner) and afterwards 'Experimental Information' in the bottom left corner. The user is now directed to the 'Experimental Information Search' section in the middle, where user can choose one of the theoretical categories in the 'Calculation Method' field (bottom arrow). In the upper 'Comments' field, the user can search the ICSD for technical details of the calculations (upper arrow). or $\mathrm{C}-\mathrm{H}$ bonds and which include structural data of pure elements, minerals, metals and intermetallic compounds; structural descriptors (Pearson symbol, ANX formula, Wyckoff sequences); and bibliographic data. In order to be included in the ICSD, a theoretical structure has to be fully characterized, the atomic coordinates determined and the composition fully specified, similarly to experimental structures. Each of the theoretical crystal structures contained in the database has been carefully evaluated and checked for quality by our expert editorial team.

\subsection{Classification and categorization of theoretical data in the ICSD}

In this section, a novel classification and categorization of theoretical data in the ICSD will be presented. Theoretical crystal structures are labelled in the ICSD to allow an easy distinction between theoretical and experimental structures (Fig. 4). The user also has the option to include all structures in a search (for a detailed description see the supporting information). Furthermore, we have defined a set of keywords which are specific for theoretical structures and which describe, for example, the methods or the details of the calculation. This ensures that the user will be able to select and evaluate those structures in a very precise manner.

In total we have defined 13 categories which correspond to the theoretical methods used to calculate theoretical crystal structures (see Table 2). Although these categories are relevant mostly for the growing field of theoretical studies, the final benefit should be for all users of the ICSD. In that respect we have suggested several theoretical categories, which are found to be most popular in the papers published with theoretical structures in the ICSD, which include the choice of the energy (cost) function, mathematical modelling, quantum chemical methods and functionals. For example, ab initio optimization, or empirical and semi-empirical potential, stands for calculations performed using the respective potentials, while geometric modelling is used when theoretical structures are obtained using mathematical and/or crystallographic models. We note that the augmented plane-wave method includes the full-potential (linearized) augmented plane-wave [(L)APW] + local orbitals (lo) method, while the linear muffin-tin orbital (LMTO) method also includes the full-potential (FP)-LMTO-atomic spheres approximation (ASA).

These theoretical categories are very useful tools for all users of the ICSD, but above all theoreticians. Possible applications span from statistics in the specific theoretical category and potential use for future calculations, to data mining and method development. In addition to 
Table 2

Summary of theoretical categories in the ICSD.

\begin{tabular}{|c|c|}
\hline Theoretical category in the ICSD & References $\dagger$ \\
\hline$A b$ initio optimization & Zagorac et al. (2014a); Mayo et al. (2016) \\
\hline Empirical and semi-empirical potential & Fan et al. (2015); Yoo et al. (2016) \\
\hline Geometric modelling & Zagorac et al. (2014b); George et al. (2015) \\
\hline Monte Carlo simulation & Hao et al. (2014); Mena et al. (2016) \\
\hline Molecular dynamics & Schmidt et al. (2015); Paściak et al. (2015) \\
\hline Plane waves method & Weerasinghe et al. (2015); Goncharov et al. (2016) \\
\hline FP(L) augmented plane-wave method $(+\mathrm{lo})$ & Mukadam et al. (2016); Cebela et al. (2017) \\
\hline Projector augmented wave method & Zurek \& Yao (2015); Buckeridge et al. (2016) \\
\hline Linear combination of atomic orbitals method & Zagorac et al. (2011); Larbi et al. (2016) \\
\hline (FP) linear muffin-tin orbital (ASA) & Uba et al. (2016); Mishra \& Ganguli (2016) \\
\hline Hartree-Fock method & Shimazaki \& Nakajima (2015); Zagorac et al. (2017a) \\
\hline Density functional theory & Civalleri et al. (2007); Schönecker et al. (2015) \\
\hline Hybrid functionals & Lee et al. (2015); Sluydts et al. (2017) \\
\hline Predicted (non-existing) crystal structure & Doll et al. (2008); Luković et al. (2017) \\
\hline Optimized (existing) crystal structure & Olsson et al. (2015); Erba et al. (2015) \\
\hline Combination of theoretical and experimental structure & Retuerto et al. (2016); Cvijović-Alagić et al. (2019) \\
\hline
\end{tabular}

$\dagger$ References to example theoretical structures found using that theoretical method and already searchable in the ICSD.

and computational materials science. Together with the previous theoretical methods, this makes in total 16 theoretical categories in the ICSD, and a complete summary of these categories is shown in Table 2.

Finally, the ICSD provides additional computational information used in the calculation of the respective theoretical crystal structures. This computational information provides details about the code, search algorithm, method, basis set information and technical details of the calculation (e.g. cutoff energy, K-point mesh etc.), providing information on reproducibility and quality of computations. In addition we provide

these 13 theoretical methods, we provide further classification and categorization based on information obtained from comparison of theoretical and experimental structures. The first such category is 'predicted (non-existing) crystal structure' (Table 2). As crystal structure predictions become more and more reliable, this category can be an excellent tool for synthesis planning. ${ }^{3}$ In particular, obtaining information on not-synthesized unknown compounds or/and not-synthesized modifications of known compounds could be an important advantage for ICSD users with numerous scientific, technological and industrial applications. The next category is 'optimized (existing) crystal structure', which compares the optimized theoretical structure with all existing experimental crystal structures in the ICSD until the year of publication. Optimized structures can also be an excellent tool for various applications: for instance, applications in computational materials science and related sciences, where optimized structures can be used to generate parameters for future calculations. In experimental materials science and related sciences, they can be used as an excellent tool for industrial and technological applications where it is very important to fine-tune materials, because slight deviations between the calculation and experiment can lead to different properties of the material. This can be even further examined by combining optimized structures with standardized keywords for physical properties. The final category is 'combination of theoretical and experimental structure'. If such data exist in the manuscript they are highly valuable to all materials scientists with a great variety of possible applications, owing to the high precision of the published data.

These categories allow comparison of calculated structures either with each other or directly with experimental data, making the categorization a useful tool in both experimental

\footnotetext{
${ }^{3}$ Of course, we note the time restriction of the predicted structures, due to the publication year. On the other hand, many theoretical structures remain nonsynthesized many years after publication.
} comments on the tolerances in energy, forces etc. used in calculations if present (which are similar to the experimental structure criteria $R$ factors, FOMs etc.). If the theoretical structure is missing the total energy criterion, meaning that the manuscript does not provide total energies, formation energies etc., the comment 'Etot ranking is missing in the paper' is added (corresponding to the ' $R$ factors are missing in the manuscript' comment). If there exist more structures in the manuscript but they are, say, energetically high and unstable, the comment 'Additional structures are published in the manuscript' is included. Furthermore, if the theoretical structure shows magnetic properties, we add comments about the magnetic state, inclusion of spin orbit interaction etc., which provide additional information on the quality of the calculation. Finally, we provide information about the code used to calculate the theoretical structure, and if additionally another code has been used, for example, for electronic property calculation or phonon calculations. Since this information is fully searchable in the ICSD, it can be a very useful tool for future theoretical studies.

\section{Applications of the ICSD}

\subsection{Discovery of new ionic conductors and solar cell} absorber

An example of using crystallographic data to predict material properties is the systematic identification of new possible $\mathrm{Na}$-ion conductors in ternary $\mathrm{Na}$ oxides for replacing $\mathrm{Li}$ in batteries. For this, Meutzner et al. $(2015,2017)$ applied the Voronoi-Dirichlet approach and were able to identify around 50 high-potential candidates for solid ionic conductors from several thousand possible structures.

The mutual influence of theoretical and experimental data in the ICSD can be illustrated by another example, where the potentially stable structure and properties of wurtzite, $\mathrm{CuGaO}_{2}$, were calculated via density functional theory (DFT) 
(Omata et al., 2014). The subsequent synthesis and analysis of the compound confirmed the expected semiconductive properties (Nagatani et al., 2015).

\subsection{Prediction of novel advanced ceramic materials}

Aluminium nitride is an interesting semiconductor ceramic material with various technological and industrial applications. In this example study, data mining of over 140000 structures in the ICSD has been performed, followed by $a b$ initio optimizations (Zagorac et al., 2017b). Finally, 12 new structure candidates were proven to be the most promising ones, which later showed diverse electronic, elastic and mechanical properties (Zagorac et al., 2018).

Similarly, transition metal silicides have attracted great attention owing to their potential applications in microelectronics, ceramics and the aerospace industry. In another example, experimental and theoretical investigations of tungsten-based silicides were performed, and new modifications were obtained using entries from the ICSD as starting points in the first principles calculations (Lukovic et al., 2017).

\subsection{Finding nature's missing binary and ternary oxide compounds}

Finding new compounds and their crystal structures is an essential step in discovering new materials. In this example, low-enthalpy phases of $\mathrm{TiO}_{2}$ and $\mathrm{SiO}_{2}$ at extreme pressure conditions were calculated using DFT. It has been found that the most stable form of $\mathrm{TiO}_{2}$ at pressures above $650 \mathrm{GPa}$ is a ten-coordinated structure with space group $I 4 / \mathrm{mmm}$. $\mathrm{TiO}_{2}$ is the well established high-pressure model for many $A X_{2}$ compounds, and this study showed that $\mathrm{SiO}_{2}$ should also form in the $I 4 / \mathrm{mmm}$ structure above $10 \mathrm{TPa}$ (Lyle et al., 2015).

In the next example a probabilistic model built on experimental data from the ICSD, novel compositions that are most likely to form a compound and their most probable crystal structures were identified and tested for stability by $a b$ initio computations. A large-scale search for new ternary oxides has been performed, which resulted in the discovery of 209 new compounds (Hautier et al., 2010).

\subsection{Structural relations studies within the ICSD}

The ICSD is a very useful tool for investigating the structural relations between various inorganic crystalline compounds. Such a study has been performed for chemical systems listed in the ICSD using a geometry-based similarity criterion. By applying all entries in the ICSD to the structure comparison algorithm CMPZ, ordered crystalline structures contained in the ICSD were classified into structure families and their relations investigated (Sultania et al., 2012). In the latter work, a hierarchical set of criteria for the separation of isopointal structures into isoconfigurational structure types has been used. It has been shown how these criteria, which include the space group, Wyckoff sequence and Pearson symbol, c/a ratio, $\beta$ ranges, ANX formulae, and, in certain cases, the necessary elements and forbidden elements, may be used to uniquely identify the representative structure types of the compounds contained in the ICSD (Schön, 2014).

\section{Conclusion}

The ICSD is already extensively used in computational and experimental materials science and related natural sciences. In particular, crystal structure predictions have become more and more reliable. This allows comparison of calculated structures either with each other or directly with experimental data. Here, we explain the introduction of theoretical CIFs into the ICSD. Each theoretical structure is extended and standardized and completely coherent with the structural standards used for experimental entries. We introduce the categorization of theoretical data in the ICSD. Finally, we present the connection of theoretical structures with material properties, applied methods and/or applications using the keyword option. This combination is an excellent tool for data mining. Therefore, the inclusion of theoretical data not only extends the scope of the ICSD significantly, it also allows data mining applications that were not possible previously while also increasing the range of data for more classical applications.

\section{Acknowledgements}

The authors thank J. C. Schön, B. Matović, D. Savić, V. Djurdjević, D. Boljanić, R. Hinek and S. Hoehler-Schlimm for collaboration and discussions.

\section{Funding information}

This work was supported by grant 45012 from the Ministry of Education, Science and Technological Development of the Republic of Serbia.

\section{References}

Allmann, R. \& Hinek, R. (2007). Acta Cryst. A63, 412-417.

Belsky, A., Hellenbrandt, M., Karen, V. L. \& Luksch, P. (2002). Acta Cryst. B58, 364-369.

Bergerhoff, G. \& Brown, I. D. (1987). Crystallographic Databases, edited by F. H. Allen, G. Bergerhoff \& R. Sievers. Chester: International Union of Crystallography.

Berman, H. M., Westbrook, J., Feng, Z., Gilliland, G., Bhat, T. N., Weissig, H., Shindyalov, I. N. \& Bourne, P. E. (2000). Nucleic Acids Res. 28, 235-242.

Buchsbaum, C., Höhler-Schlimm, S. \& Rehme, S. (2010). Data Mining in Crystallography, edited by D. Hofmann \& L. N. Kuleshova. Heidelberg: Springer Verlag.

Buckeridge, J., Jevdokimovs, D., Catlow, C. R. A. \& Sokol, A. A. (2016). Phys. Rev. B, 93, 125205.

Butler, K. T., Frost, J. M., Skelton, J. M., Svane, K. L. \& Walsh, A. (2016). Chem. Soc. Rev. 45, 6138-6146.

Čebela, M., Zagorac, D., Batalović, K., Radaković, J., Stojadinović, B., Spasojević, V. \& Hercigonja, R. (2017). Ceram. Int. 43, 1256-1264. Civalleri, B., Doll, K. \& Zicovich-Wilson, C. M. (2007). J. Phys. Chem. $B, \mathbf{1 1 1}, 26-33$.

Curtarolo, S., Hart, G. L. W., Nardelli, M. B., Mingo, N., Sanvito, S. \& Levy, O. (2013). Nat. Mater. 12, 191-201. 
Curtarolo, S., Setyawan, W., Wang, S., Xue, J., Yang, K., Taylor, R. H., Nelson, L. J., Hart, G. L. W., Sanvito, S., Buongiorno-Nardelli, M., Mingo, N. \& Levy, O. (2012). Comput. Mater. Sci. 58, 227-235.

Cvijović-Alagić, I., Cvijović, Z., Zagorac, D. \& Jovanović, M. T. (2019). Ceram. Int. 45, 9423-9438.

Doll, K., Schön, J. C. \& Jansen, M. (2008). Phys. Rev. B, 78, 144110. Downs, R. T. \& Hall-Wallace, M. (2003). Am. Mineral. 88, 247-250.

Draxl, C. \& Scheffler, M. (2018). MRS Bull. 43, 676-682.

Erba, A., Ruggiero, M. T., Korter, T. M. \& Dovesi, R. (2015). J. Chem. Phys. 143, 144504

Fan, Q., Wang, C., Yu, T. \& Du, J. (2015). Physica B, 456, 283-292.

George, J., Deringer, V. L. \& Dronskowski, R. (2015). Inorg. Chem. 54, 956-962.

Goncharov, A. F., Lobanov, S. S., Kruglov, I., Zhao, X., Chen, X., Oganov, A. R., Konôpková, Z. \& Prakapenka, V. B. (2016). Phys. Rev. B, 93, 174105.

Gražulis, S., Daškevič, A., Merkys, A., Chateigner, D., Lutterotti, L., Quirós, M., Serebryanaya, N. R., Moeck, P., Downs, R. T. \& Le Bail, A. (2012). Nucleic Acids Res. 40, D420-D427.

Groom, C. R., Bruno, I. J., Lightfoot, M. P. \& Ward, S. C. (2016). Acta Cryst. B72, 171-179.

Hall, S. R. (1991). J. Chem. Inf. Model. 31, 326-333.

Hall, S. R. \& Spadaccini, N. (1994). J. Chem. Inf. Model. 34, 505-508.

Hao, S., Zhao, L., Chen, C., Dravid, V. P., Kanatzidis, M. G. \& Wolverton, C. M. (2014). J. Am. Chem. Soc. 136, 1628-1635.

Hautier, G., Fischer, C. C., Jain, A., Mueller, T. \& Ceder, G. (2010). Chem. Mater. 22, 3762-3767.

ICDD (2018). PDF-4+ 2019. International Centre for Diffraction Data, Newtown Square, PA, USA.

Jain, A., Ong, S. P., Hautier, G., Chen, W., Richards, W. D., Dacek, S., Cholia, S., Gunter, D., Skinner, D., Ceder, G. \& Persson, K. A. (2013). APL Mater. 1, 011002.

Káňa, T., Hüger, E., Legut, D., Čák, M. \& Šob, M. (2016). Phys. Rev. $B, \mathbf{9 3}, 134422$.

Larbi, T., Doll, K. \& Manoubi, T. (2016). J. Alloys Compd. 688, 692698.

Lee, H., Cheong, S. W. \& Kim, B. G. (2015). J. Solid State Chem. 228, 214-220.

Luković, J., Zagorac, D., Schön, J. C., Zagorac, J., Jordanov, D., Volkov-Husović, T. \& Matović, B. (2017). Z. Anorg. Allg. Chem. 643, 2088-2094.

Lyle, M. J., Pickard, C. J. \& Needs, R. J. (2015). Proc. Natl Acad. Sci. USA, 112, 6898-6901.

Mayo, M., Griffith, K. J., Pickard, C. J. \& Morris, A. J. (2016). Chem. Mater. 28, 2011-2021.

Mena, J. M., Schoberth, H., Gruhn, T. \& Emmerich, H. (2016). Acta Mater. 111, 157-165.

Meutzner, F., Münchgesang, W., Kabanova, N. A., Zschornak, M., Leisegang, T., Blatov, V. A. \& Meyer, D. C. (2015). Chem. Eur. J. 21, 16601-16608.

Meutzner, F., Münchgesang, W., Leisegang, T., Schmid, R., Zschornak, M., Urena de Vivanco, M., Shevchenko, A. P., Blatov, V. A. \& Meyer, D. C. (2017). Cryst. Res. Technol. 52, 1600223.

Miao, M., Botana, J., Zurek, E., Hu, T., Liu, J. \& Yang, W. (2016). Chem. Mater. 28, 1994-1999.

Mishra, S. \& Ganguli, B. (2016). Mater. Chem. Phys. 173, 429-437.
Mukadam, M. D., Roy, S., Meena, S. S., Bhatt, P. \& Yusuf, S. M. (2016). Phys. Rev. B, 94, 214423.

Nagatani, H., Suzuki, I., Kita, M., Tanaka, M., Katsuya, Y., Sakata, O., Miyoshi, S., Yamaguchi, S. \& Omata, T. (2015). Inorg. Chem. 54, 1698-1704.

NIMS (2018). AtomWorks-Adv. National Institute for Materials Science, Tsukuba, Ibaraki, Japan.

Olsson, P. A. T., Blomqvist, J., Bjerkén, C. \& Massih, A. R. (2015). Comput. Mater. Sci. 97, 263-275.

Omata, T., Nagatani, H., Suzuki, I., Kita, M., Yanagi, H. \& Ohashi, N. (2014). J. Am. Chem. Soc. 136, 3378-3381.

Ortiz, C., Eriksson, O. \& Klintenberg, M. (2009). Comput. Mater. Sci. 44, 1042-1049.

Paściak, M., Welberry, T. R., Heerdegen, A. P., Laguta, V., Ostapchuk, T., Leoni, S. \& Hlinka, J. (2015). Phase Transit. 88, 273-282.

Retuerto, M., Skiadopoulou, S., Li, M., Abakumov, A. M., Croft, M., Ignatov, A., Sarkar, T., Abbett, B. M., Pokorný, J., Savinov, M., Nuzhnyy, D., Prokleška, J., Abeykoon, M., Stephens, P. W., Hodges, J. P., Vaněk, P., Fennie, C. J., Rabe, K. M., Kamba, S. \& Greenblatt, M. (2016). Inorg. Chem. 55, 4320-4329.

Saal, J. E., Kirklin, S., Aykol, M., Meredig, B. \& Wolverton, C. (2013). JOM, 65, 1501-1509.

Schmidt, K. M., Buettner, A. B., Graeve, O. A. \& Vasquez, V. R. (2015). J. Mater. Chem. C, 3, 8649-8658.

Schön, J. C. (2014). Z. Anorg. Allg. Chem. 640, 2717-2726.

Schönecker, S., Li, X., Koepernik, K., Johansson, B., Vitos, L. \& Richter, M. (2015). RSC Adv. 5, 69680-69689.

Shimazaki, T. \& Nakajima, T. (2015). J. Chem. Phys. 142, 074109.

Sluydts, M., Pieters, M., Vanhellemont, J., Van Speybroeck, V. \& Cottenier, S. (2017). Chem. Mater. 29, 975-984.

Sultania, M., Schön, J. C., Fischer, D. \& Jansen, M. (2012). Struct. Chem. 23, 1121-1129.

Uba, S., Bonda, A., Uba, L., Bekenov, L. V., Antonov, V. N. \& Ernst, A. (2016). Phys. Rev. B, 94, 054427.

Villars, P. \& Cenzual, K. (2018). Pearson's Crystal Data: Crystal Structure Database for Inorganic Compounds (on DVD), Release 2018/19. ASM International, Materials Park, Ohio, USA.

Weerasinghe, G. L., Pickard, C. J. \& Needs, R. J. (2015). J. Phys. Condens. Matter, 27, 455501.

White, P. S., Rodgers, J. R. \& Le Page, Y. (2002). Acta Cryst. B58, $343-$ 348 .

Yoo, S.-H., Lee, J.-H., Jung, Y.-K. \& Soon, A. (2016). Phys. Rev. B, 93, 035434.

Zagorac, D., Doll, K., Schön, J. C. \& Jansen, M. (2011). Phys. Rev. B, 84, 045206.

Zagorac, D., Doll, K., Zagorac, J., Jordanov, D. \& Matović, B. (2017a). Inorg. Chem. 56, 10644-10654.

Zagorac, D., Schön, J. C., Zagorac, J. \& Jansen, M. (2014a). Phys. Rev. $B, \mathbf{8 9}, 075201$.

Zagorac, J., Zagorac, D., Jovanović, D., Luković, J. \& Matović, B. (2018). J. Phys. Chem. Solids, 122, 94-103.

Zagorac, J., Zagorac, D., Rosić, M., Schön, J. C. \& Matović, B. (2017b). CrystEngComm, 19, 5259-5268.

Zagorac, J., Zagorac, D., Zarubica, A., Schön, J. C., Djuris, K. \& Matovic, B. (2014b). Acta Cryst. B70, 809-819.

Zurek, E. \& Yao, Y. (2015). Inorg. Chem. 54, 2875-2884. 